\title{
Dopamine Fluctuations in the Nucleus Accumbens during Maintenance, Extinction, and Reinstatement of Intravenous D-Amphetamine Self-Administration
}

\author{
Robert Ranaldi, Dorothy Pocock, Richard Zereik, and Roy A. Wise \\ Center for Studies in Behavioral Neurobiology, Concordia University, Montreal, Québec, Canada H3G 1M5
}

\begin{abstract}
Moment-to-moment fluctuations of nucleus accumbens dopamine (DA) were determined in rats self-administering or passively receiving "yoked" intravenous infusions of D-amphetamine. The initial lever presses of each session caused elevations in DA concentration, usually to an initial peak that was not maintained throughout the rest of the session. As the initial ("loading") injections were metabolized, DA levels dropped toward baseline but were sustained at elevated plateaus by subsequent lever pressing that was spaced throughout the remainder of the $3 \mathrm{hr}$ sessions. During this period, DA levels fluctuated phasically, time-locked to the cycle of periodic lever pressing. Consistent with the known pharmacological actions and dynamics of amphetamine, peak DA elevations were seen $\sim 10-15 \mathrm{~min}$ after each injection, and the mean DA level was at a low point in the phasic cycle at the time of each new lever press. During extinction periods when saline was substituted for amphet-
\end{abstract}

Laboratory animals will learn arbitrary response habits such as lever pressing when intravenous injections of the psychomotor stimulants amphetamine or cocaine are made contingent on such responses. Within a few weeks of regular testing the behavior stabilizes, appearing to become regulated by the systemic drug level. After responding rapidly for the first minutes (the "loading" phase) of a session, experienced animals settle into a pattern of spaced responding ("maintenance" phase) that is predictable from the half-life of the rewarding drug (Dougherty and Pickens, 1974). In experienced animals, decreases in dose or increases in response requirement-over the ranges of these parameters that sustain regular responding — are met with compensatory increases in response rate such that relatively constant hourly drug intake is maintained (Pickens and Thompson, 1968). Regardless of the unit dose within this range, rats respond for the next D-amphetamine injection when blood levels fall to $\sim 0.2 \mu \mathrm{g} / \mathrm{ml}$ (Yokel and Pickens, 1974). This apparent regulation is seen even when the unit dose is varied unpredictably within sessions; in this case, the time until the next response is closely related to the amount given in the previous injection (Gerber and Wise, 1989).

\footnotetext{
Received Dec. 11, 1998; revised Feb. 18, 1999; accepted Feb. 24, 1999.

This research was supported by grants from the Medical Research Council of Canada, the National Institute on Drug Abuse of the United States (DA1720), and Fonds pour la Formation de Chercheurs et l'Aide à la Recherche (Québec). We wish to thank Zafiro Koty for excellent technical assistance.

Correspondence should be addressed to Dr. Robert Ranaldi, Division of Neurobiology and Behavior Research, Department of Psychiatry and Human Behavior, University of Mississippi Medical Center, 2500 North State Street, Jackson, MS 39216.

Dr. Wise's present address: Intramural Research Program, National Institute on Drug Abuse, Rockville, MD 20857.

Copyright (C) 1999 Society for Neuroscience 0270-6474/99/194102-08\$05.00/0
}

amine, DA levels dropped steadily toward baseline levels despite a dramatic increase in (now-unrewarded) lever pressing. Noncontingent injections during extinction reinstated leverpressing behavior and increased nucleus accumbens DA concentrations. These data are consistent with the hypothesis that under the conditions of this experiment-during periods of amphetamine intoxication in well-trained animals-the timing of amphetamine self-administration comes primarily under the control of extracellular DA concentrations. The probability of lever pressing during the maintenance phase is highest when DA concentrations fall near a characteristic trigger point, a trigger point that is significantly elevated above baseline, and falls as DA concentrations fall below or increase above that trigger point.

Key words: amphetamine; self-administration; microdialysis; reward; rats; extinction; reinstatement
The mechanism of this apparent regulation of intake, which is seen only in experienced animals and only when they are already intoxicated, is not clearly understood. It is presumed that some common neural consequence of amphetamine and cocaine [and perhaps other drugs of abuse (Wise and Bozarth, 1987)] determines the probability of responding after the animal becomes initially intoxicated. Because it is the ability of each of these drugs to elevate extracellular dopamine concentrations in the nucleus accumbens that seems critical for their rewarding effects (Yokel and Wise, 1975), it has been of considerable interest to determine whether the timing of drug-seeking responses is predictable from fluctuations in extracellular dopamine concentrations (Wise, 1993). Initial in vivo voltammetry (Gratton and Wise, 1994) and microdialysis (Wise et al., 1995b) studies each suggest that it is.

In the present study we used microdialysis to examine fluctuations of nucleus accumbens dopamine during intravenous amphetamine self-administration. Although amphetamine selfadministration was expected to produce dopamine fluctuations qualitatively similar to those seen with cocaine, two differences between the two drugs make amphetamine self-administration particularly interesting. First, cocaine does not cause dopamine release but rather prolongs the extracellular half-life of dopamine released as a consequence of dopaminergic impulse flow (Heikkila et al., 1975a). Amphetamine, on the other hand, can cause impulse-independent dopamine release, and thus its ability to elevate extracellular dopamine does not depend critically on other inputs to the dopamine system (Heikkila et al., 1975b). Because there is reward-related synaptic input to the dopamine system (Schultz, 1997), this difference in the mechanism of action makes comparison of the two drugs of particular interest. Second, 
rats respond considerably less often for moderate doses of intravenous amphetamine than they do for moderate doses of intravenous cocaine. Thus the limits of temporal resolution inherent in microdialysis measurements should be less troublesome in the characterization of dopaminergic fluctuations during amphetamine self-administration. The slow rate of amphetamine selfadministration allowed for the collection of approximately five or six microdialysis samples ( 5 min each; $20 \mu \mathrm{l}$ each) between each injection, allowing us to estimate the pattern of DA fluctuations with both high resolution and higher accuracy than in cocaine self-administration studies (Wise et al., 1995b) in which responding is five to six times faster and 1 min microdialysis samples must be taken if between-injection fluctuations are to be detected. We also investigated DA concentrations in animals receiving unearned or "yoked" infusions of D-amphetamine and in animals responding during periods of extinction, both in the absence of and in the presence of experimenter-administered ("priming") injections.

\section{MATERIALS AND METHODS}

Subjects and surgery. Subjects were 18 male Long Evans rats (Charles River Laboratories, Wilmington, MA) weighing between 350 and $400 \mathrm{gm}$ at the time of surgery. All subjects had access to food (Purina rat chow) and water ad libitum except during self-administration sessions. Each rat was implanted, under sodium pentobarbital $(65 \mathrm{mg} / \mathrm{kg}$, i.p.), with a guide cannula and a permanently indwelling jugular catheter. The 20-gauge stainless steel guide cannulae were angled toward the midline, aimed at the nucleus accumbens [incisor bar $5 \mathrm{~mm}$ above the interaural line, anteroposterior $3.4 \mathrm{~mm}$, mediolateral $2.5 \mathrm{~mm}$, and dorsoventral $-4.3 \mathrm{~mm}$ along the angled cannula track (Pellegrino et al., 1979)]. The assembly was anchored to the skull and to four stainless steel skull screws with dental acrylic. Stainless steel obturators (28-gauge wires, flush with the tips of the guide cannulae) were inserted at the time of surgery and remained there until $3 \mathrm{~d}$ before the microdialysis test session. At this time they were replaced with obturators that extended $4 \mathrm{~mm}$ beyond the ends of the guide cannulae, to the depth the microdialysis probe would subsequently reach.

For each rat an incision was made in the neck, and the jugular vein was isolated and opened. A SILASTIC intravenous catheter (Dow Corning, Midland, MI) was inserted into the vein to a depth just short of the right atrium. The other end of the catheter was fed subcutaneously to the back of the neck. There, a piece of bent 22-gauge stainless steel tubing was inserted into the end of the catheter and secured with dental acrylic to the cannula assembly. The tubing was capped when not in use. The catheter was flushed with a heparin-saline solution (200 USP) immediately after surgery and daily thereafter.

Amphetamine self-administration. Amphetamine self-administration training began the day after surgery. The animals were placed in $26 \mathrm{~cm} \times$ $26 \mathrm{~cm}$ operant chambers and tested in daily $4 \mathrm{hr}$ sessions. The chambers were equipped with a $2.5 \mathrm{~cm}$ lever mounted $10 \mathrm{~cm}$ above the grid floors on the rear wall. A white cue light was mounted $3 \mathrm{~cm}$ above the lever. Each rat was connected by polyethylene tubing, through one channel of a dual channel swivel, to syringes in individual syringe pumps. Each lever press activated the syringe pump and cue light for $28 \mathrm{sec}$ during which lever presses were counted but had no other scheduled consequences. The pump activation resulted in an infusion of D-amphetamine sulfate $(0.25 \mathrm{mg} / \mathrm{kg})$ in $0.25 \mathrm{ml}$ of physiological saline.

Microdialysis probes. Removable concentric microdialysis probes were constructed according to the procedures developed by Robinson and Whishaw (1988). Each probe consisted of a polyethylene tubing (PE20) fluid inlet attached to a 26-gauge stainless steel outer cannula. This cannula was cemented to $4 \mathrm{~mm}$ of cellulose dialysis fiber [Spectrum membrane from Fisher Scientific, Houston, TX; inner diameter (i.d.) = $215 \mu \mathrm{m}$; outer diameter (o.d.) $=251 \mu \mathrm{m} ; 13,000$ molecular weight cutoff] that was sealed at the end with a $1 \mathrm{~mm}$ epoxy cement plug. The fluid outlet was capillary silica tubing (Polymicro Technologies, Phoenix, AZ; i.d. $=75 \mu \mathrm{m}$; o.d. $=150 \mu \mathrm{m})$ that extended $3 \mathrm{~mm}$ into the dialysis fiber. The silica outlet tubing exited the inlet polyethylene tubing through a sealed cut near the animal's head (sealed with epoxy cement) and terminated in a collection vial $25 \mathrm{~cm}$ above the probe.

Microdialysis testing. Microdialysis samples were collected from three groups of rats. The experienced-self-administering (-SA) group $(n=8)$ consisted of rats that had been trained to self-administer D-amphetamine and were engaged in self-administration during microdialysis testing. The experienced-yoked group $(n=4)$ consisted of rats that had been trained to self-administer amphetamine but, during microdialysis testing, passively received injections as the yoked partner of a self-administering rat. The inexperienced-yoked group $(n=4)$ consisted of rats that were naïve to self-administration and D-amphetamine and, during microdialysis testing, received unearned intravenous injections of amphetamine as the yoked partner of a self-administering rat.

Approximately $18 \mathrm{hr}$ before a given microdialysis test session, the animal was implanted with a microdialysis probe. On some occasions it was necessary to anesthetize the rats briefly with sodium methohexital (100 $\mu$ l, i.v.) before the probe was inserted. After the microdialysis probes were implanted, the rats remained in their test chambers until the end of the test session. Artificial CSF (aCSF; $\mathrm{Na}^{+}, 145 \mathrm{~mm} ; \mathrm{K}^{+}, 2.7 \mathrm{~mm}$; $\mathrm{Mg}^{2+}, 1.0 \mathrm{~mm}$; $\mathrm{Ca}^{2+}, 1.2 \mathrm{mM} ; \mathrm{Cl}^{-}, 150 \mathrm{~mm}$; and ascorbate, $0.2 \mathrm{~mm}$ in 2.0 $\mathrm{mm}$ Sorensens phosphate buffer at $\mathrm{pH}$ 7.4) was perfused through the probe at a rate of $0.2 \mu \mathrm{l} / \mathrm{min}$ during the waiting period and at $2.0 \mu \mathrm{l} / \mathrm{min}$ during the test session. During the waiting period the levers and cue lights were hidden by cage partitions, and the rats had access to food and water ad libitum. During the microdialysis test session, 10 or $15 \mathrm{~min}$ baseline dialysate samples were collected for a period of $60-90 \mathrm{~min}$. After the final baseline sample had been collected, the rats were carefully lifted out of the operant chambers and handled for a few seconds (to mimic partially the start of a regular self-administration session). At this time the partitions hiding the levers and cue lights were removed in the case of the experienced-SA rats. After returning to the chambers, rats having access to the levers were allowed to self-administer drug, and the remaining rats began receiving yoked injections.

Microdialysis samples were taken at $5 \mathrm{~min}$ intervals from the experienced-SA group during a $3 \mathrm{hr}$ self-administration session (in the case of each of two rats, DL113 and DL146, samples collected during the last 30 min of the session were ruined during the HPLC procedure). At the end of this self-administration session, the amphetamine-filled syringe was removed from the pump so that lever presses would continue to activate the pump and cue light but fail to result in drug delivery. For $4 \mathrm{hr}$, the animals were allowed to lever press in this extinction period; in this phase, when dopamine levels were no longer elevated by amphetamine, 10 min dialysis samples were taken. At the end of the extinction period, the amphetamine-filled syringes were replaced, and each rat was administered a noncontingent (priming) injection of D-amphetamine and allowed to resume self-administration. During this "reinstatement" period, 5 min dialysate samples were again collected.

Microdialysis samples were also taken at $5 \mathrm{~min}$ intervals from the experienced- and inexperienced-yoked groups; these groups were given yoked injections for $3 \mathrm{hr}$. For the experienced-yoked group, the injection patterns were randomly chosen from a set of recorded selfadministration patterns belonging to the yoked animal itself. For the inexperienced-yoked group, the injection patterns were randomly chosen from a set of recorded self-administration patterns belonging to another animal. There were no extinction or reinstatement periods for experienced- and inexperienced-yoked groups.

All dialysate samples were collected directly into mobile phase (a strong antioxidant) and placed immediately on dry ice until the end of the test session when they were placed in an ultracold environment $\left(-80^{\circ} \mathrm{C}\right)$ where they were kept until they were analyzed.

Dopamine assay. Dialysate samples were assayed for DA using reverse phase, isocratic, ion-pairing, HPLC with electrochemical detection in the redox mode. Frozen dialysate samples were thawed, vortexed, and loaded into a refrigerated autoinjector (Spectra System AS3500; Thermo Separation Products) that maintained a temperature of $1^{\circ} \mathrm{C}$ during the analytical run. Samples were injected onto a $15 \mathrm{~cm} \times 4.6 \mathrm{~mm}$ C18 column (CSC-Sil 80A/ODS2, $5 \mu \mathrm{m}$; Chromatography Sciences, St. Laurent, Québec, Canada) or, during the later phase of the study, a $5 \mathrm{~cm} \times 4.6 \mathrm{~mm}$ C18 column (Supelcosil LC-18, $3 \mu \mathrm{m}$; Supelco, Toronto, Ontario, Canada) by way of a Rheodyne injection valve with a $100 \mu$ l loop. Mobile phase $(0.06 \mathrm{M}$ sodium phosphate monobasic, $0.03 \mathrm{M}$ citric acid, $0.035 \mathrm{~mm}$ SDS, $0.1 \mathrm{~m}$ EDTA, and 25\% HPLC-grade methanol in nanopure water; $\mathrm{pH}$-adjusted to 3.35 with $1 \mathrm{~N}$ sodium hydroxide) was recycled at 1.2 $\mathrm{ml} / \mathrm{min}$ by a Hitachi pump (model L-7100).

DA was quantified by the use of an ESA Coulochem II Detector (model 5200) with two analytical electrodes: an oxidizing electrode $(+340 \mathrm{mV})$ and a reducing electrode $(-300 \mathrm{mV})$. The sensitivity of the reducing electrode was 50-100 times greater than the sensitivity of the 
Experienced-SA group
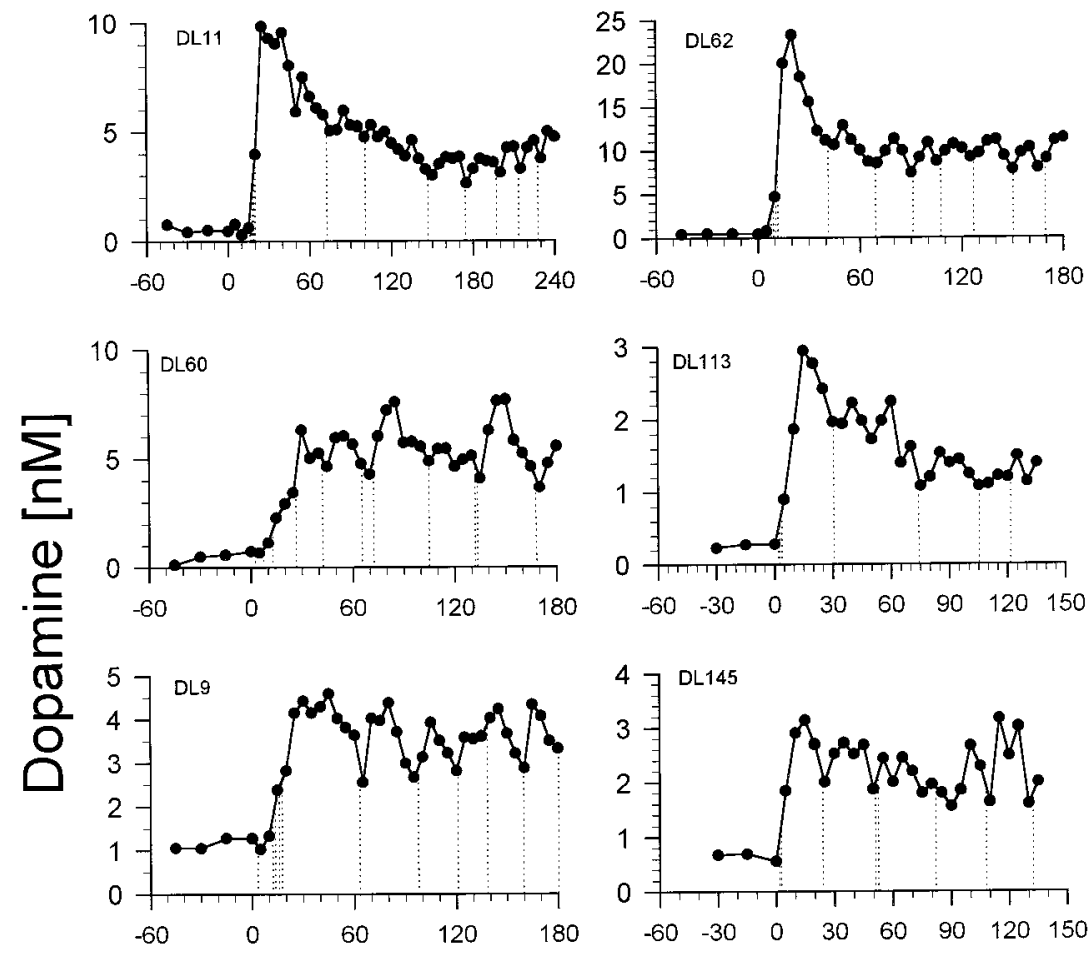

Figure 1. Nucleus accumbens dopamine concentrations in rats self-administering intravenous doses $(0.25 \mathrm{mg} / \mathrm{kg}$ per injection) of D-amphetamine. Dialysate samples were collected at $15 \mathrm{~min}$ intervals before the start of the self-administration session at time $=0$ (baseline) and at 5 min intervals after the start of the session. Vertical dotted lines represent lever presses and associated $\mathrm{D}$-amphetamine infusions. $D L$ numbers are rat identification numbers.
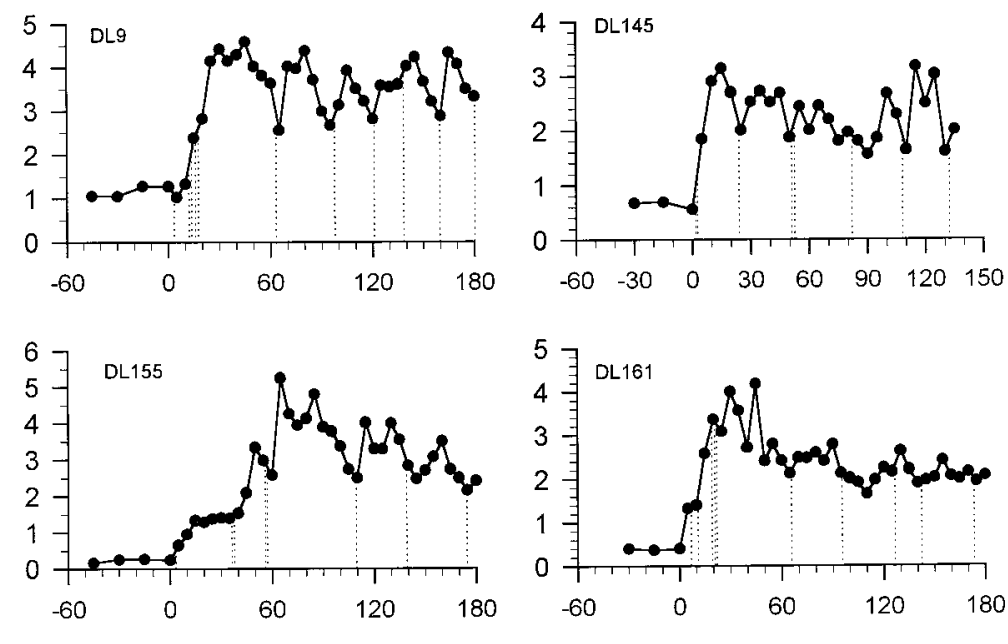

Time [min]

oxidizing electrode. (The full-scale response window at the reducing electrode was $10 \mathrm{nA}$ with the longer column and $5 \mathrm{nA}$ with the shorter column.) Output from the analytical electrodes went to a dual-channel integrator (model SP4200; Spectra-Physics, Fremont, CA) connected to Spectra-Physics Winner software on a 486 IBM-compatible computer. The reducing electrode was connected to the channel programmed to integrate the areas under the DA peak, and the oxidizing electrode was connected to the channel programmed to integrate areas under the peaks of DA metabolites. The system was calibrated with standards (Sigma, St. Louis, MO) dissolved in aCSF diluted with mobile phase in the same ratio that was used for the samples.

The approximate retention times of DOPAC, 5-hydroxyindolacetic acid, 4-hydroxy-3-methoxyphenylacetic acid, and DA were 2, 3, 4, and 6-7 min, respectively, on the longer column and 1.5, 2.0, 3.0, and 3.4-4.5 min, respectively, on the shorter column.

Histology. After microdialysis testing, the rats were anesthetized with sodium pentobarbital, perfused with saline followed by $10 \%$ formalin, and decapitated. Their brains were removed and stored in $10 \%$ formalin for at least $7 \mathrm{~d}$ before being cut in $40 \mu \mathrm{m}$ serial sections and inspected for probe implantation sites.

Data analysis. Only the HPLC results for dialysate samples collected during the period of steady-state responding (maintenance phase) after the first hour of the session were used for calculating means and for statistical analyses. For statistical analyses all data were transformed into the percentage of baseline values to minimize the between-animal variability. Means were calculated using the percentage of baseline values. The DA values for the samples during which lever presses occurred were averaged. This calculation was performed for each of the four samples occurring before and after lever presses. Thus, for each animal, we calculated a mean value for the fourth, third, second, and first samples taken before a lever press, the mean value for the samples during which a lever press occurred, and the mean value for the first, second, third, and fourth samples taken after a lever press. These means were used in a two-factor ANOVA with sample number as a repeated-measures factor.

\section{RESULTS}

Each rat given the opportunity to self-administer D-amphetamine learned to do so within 3-10 d, developing a stable pattern of intake within $14 \mathrm{~d}$. Typically, the rats self-administered three to six infusions in the first 30 min of a session (loading phase) and then maintained a regular intake of approximately two or three infusions per hour throughout the remainder (maintenance phase) of the session (Fig. 1). Baseline concentrations of dopamine varied between 0.25 and $2.5 \mathrm{~nm}$. Each rat initiated lever pressing within minutes of being replaced in its chamber with the lever exposed. The inf usions taken during the loading phase were accompanied by increases in dopamine concentration ranging from 4 - to $>20$-fold. The end of the loading phase was typically marked by a pause in lever pressing that lasted from 25 to $50 \mathrm{~min}$ and that was accompanied by a steady decrease in dopamine concentrations. Dopamine concentrations did not fall to baseline levels, however, but were maintained (by the animal's resumption 


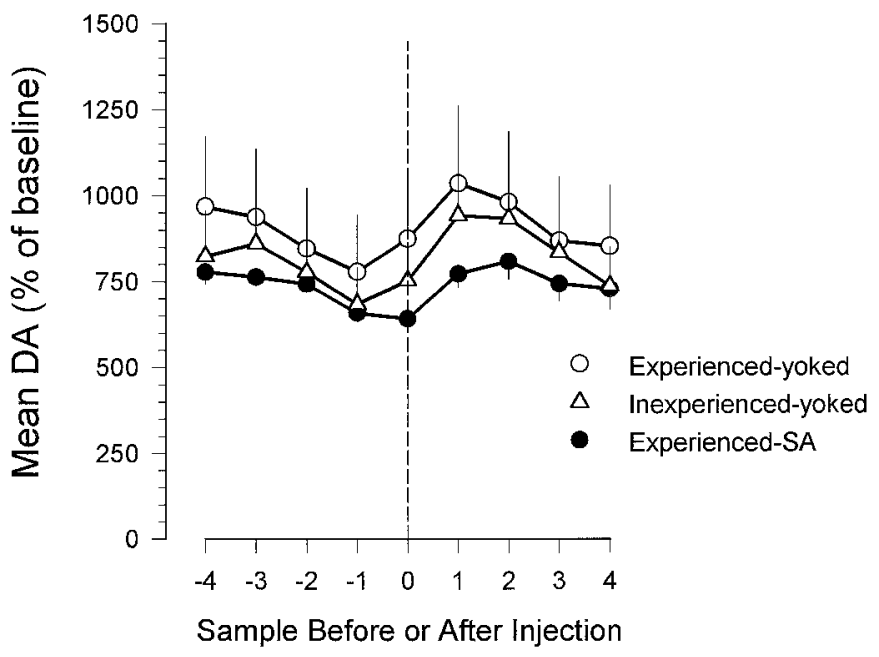

Figure 2. Mean elevations over baseline in nucleus accumbens dopamine concentrations in rats receiving self-administered or experimenteradministered intravenous injections of D-amphetamine. For each rat the mean dopamine concentration was calculated for the four samples before and after an injection occurred as well as for the sample during which the injection occurred. Only the results for dialysate samples collected after the first $60 \mathrm{~min}$ of the session were used. These data were then averaged within each group. The vertical dashed line represents the time of a D-amphetamine infusion.

of lever pressing) at substantially elevated values for as long as the drug remained available. Responding was sustained throughout the maintenance phase, and inter-response times in this period were relatively uniform (Fig. 1).

Although DA concentrations remained tonically elevated during the maintenance phase, they fluctuated phasically around elevated plateaus as a function of the self-administered infusions (Fig. 1). The mean dopamine concentration usually rose in the first and second periods after an infusion and fell in the four sample periods before the next infusion (Fig. 2). Thus peak dopamine concentrations occurred between 10 and 15 min after infusions, and the lowest dopamine concentrations occurred in the samples collected at the times of lever pressing.

Rats in the experienced-and inexperienced-yoked groups (the groups that received unearned and unsignaled infusions of D-amphetamine) showed rise-and-fall dopamine profiles similar in extent and time course to those of the rats in the experienced-SA group (the group that self-administered the drug) (Fig. 3). Dopamine concentrations in these animals were sustained at tonically elevated plateaus, as in the self-administering rats, throughout the sessions, tending to phasically decrease before and increase after each infusion. The increases over baseline in dopamine concentrations seen in the experienced- and inexperienced-yoked groups were somewhat greater than those seen in the experienced-SA group (Fig. 2; a two-way ANOVA revealed significant time $\left[F_{(7,91)}=11.73 ; p<0.001\right]$ and group $\left[F_{(2,13)}=\right.$ $27.40 ; p<0.001]$ effects but no significant time $\times$ group interaction; Scheffé tests on the group factor revealed that the three groups were significantly different from each other $[p<0.05])$.

The extinction period was associated with dramatic increases in lever pressing. The four rats tested under this condition pressed their levers 359, 306, 105, and 147 times. The highest response rates occurred during the first $2 \mathrm{hr}$ of extinction. Despite having increased lever pressing, the extinction period was associated with a progressive decline in dopamine concentrations to or below baseline levels (Fig. 4). The greatest decline in dopamine concentrations tended to coincide with the period of highest lever pressing (first $2 \mathrm{hr}$ of extinction). During the final 30 or more minutes of extinction, all rats had ceased stereotyped sniffing or locomotion and were lying down. A single noncontingent infusion of D-amphetamine caused the rats to reinitiate the exploratory locomotion and sniffing that is typically seen under the influence of this drug. In three of the four rats, the noncontingent infusion resulted in an immediate increase in dopamine concentration followed a few minutes later by the resumption of lever pressing for the drug (see Fig. 4). In the case of DL80, resumption of lever pressing occurred before it was possible to observe increases in dopamine concentrations.

Histological inspection revealed all microdialysis probes to be situated within the nucleus accumbens. The majority of placements were in the shell region of the nucleus, approximately in the middle of its rostral-caudal extent (Fig. 5).

\section{DISCUSSION}

Dopamine levels were tonically elevated throughout the period of drug self-administration, increasing after the first injection, reaching a peak within the first several injections, dropping to an elevated plateau for as long as drug remained available, falling back to baseline during extinction, and elevating again with priming and renewed drug availability. Phasic fluctuations were seen during the maintenance phase of the self-administration sessions, when responding was regular and is known to result in the maintenance of nearly constant hourly drug intake despite changes in the dose per injection (Yokel and Pickens, 1974) or, within limits, the work requirements (Pickens and Thompson, 1971).

During the maintenance phase, dopamine levels were elevated after each injection, usually within the first dialysis sample after the injection. Dopamine levels fell again, reaching approximately the same characteristic trigger point for a given animal just before the next lever press. Although the approximate trigger points for different animals varied greatly, it is not clear whether this is a characteristic of the animal or of the probe placement or characteristics. It cannot be determined from the present study whether the relative constancy of the dopamine concentration at the time of lever pressing in the maintenance phase was a cause or a consequence of the regularity of response rate. However, in a cocaine self-administration study in which dose was varied from one injection to the next and caused response times to vary accordingly, it was dopamine concentration in the nucleus accumbens rather than time since the last injection that predicted the time of the next response (Wise et al., 1995b). Thus it seems likely that the rate of responding is under the control of some correlate of dopamine concentration and not merely under the control of a timed response habit. The most interesting possibility from our own point of view is that additional amphetamine apparently has little if any rewarding efficacy when dopamine levels are already elevated much beyond the trigger point for a given animal. This suggests some form of saturation in the reward system, corresponding to periods of drug "satiety" in which additional drug does not cause any immediate increment in reward intensity. Although additional drug will prolong the experience of reward, it appears that it adds no immediate reward increment to which the animal is responsive.

The pattern of dopamine fluctuations in rats self-administering amphetamine (present data) is similar to the pattern observed in rats self-administering cocaine (Wise et al., 1995b) except for the 


\section{Experienced-yoked}
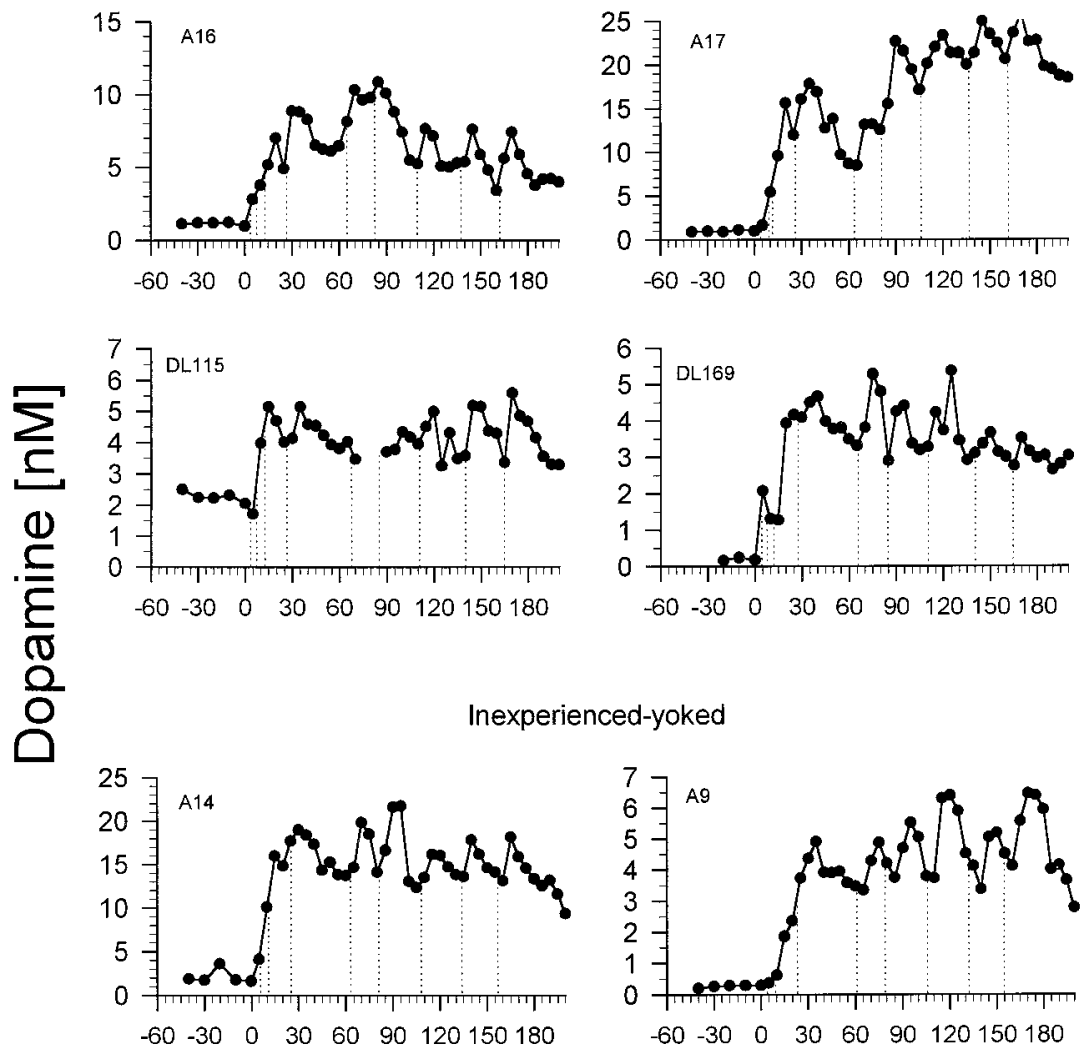

Figure 3. Nucleus accumbens dopamine concentrations in rats receiving experimenter-administered intravenous doses of D-amphetamine $(0.25 \mathrm{mg} / \mathrm{kg}$ per injection). Top, These four rats (experienced-yoked) had a history of amphetamine self-administration. Bottom, These four rats (inexperienced-yoked) were naïve to amphetamine and self-administration. Dialysate samples were collected at 10 min intervals before the start of the session at time $=0$ (baseline) and at $5 \mathrm{~min}$ intervals after the start of the session. Vertical dotted lines represent the receipt of D-amphetamine infusions. $A$ and $D L$ numbers are rat identification numbers.
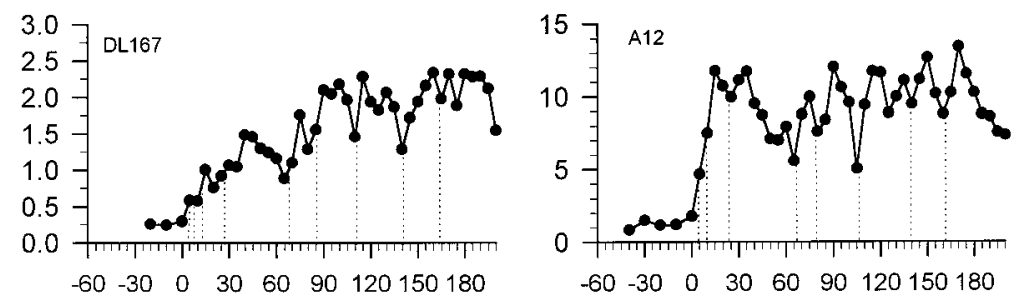

Time [min]

longer cycle time - which can be expected considering its longer time of onset and half-life - in the case of amphetamine. The similarity between the dopamine fluctuation patterns of the two drugs suggests that, despite differences in their direct neurochemical effects on the dopamine system, the two drugs have very similar actions as reinforcers. Cocaine, unlike amphetamine, does not cause the release of dopamine but merely blocks its reuptake (Heikkila et al., 1975a; Berger et al., 1990). The ability of cocaine to elevate nucleus accumbens dopamine levels thus depends much more than does that of amphetamine on impulse flow in the dopamine system. Because impulse flow in the dopamine system may be caused by synaptic input related to rewarding events (Schultz, 1997), it might be expected that the different mechanisms of action on dopamine of the two drugs would result in somewhat different dopamine profiles during rewarding drug selfadministration. However, no such difference was obvious.

Studies involving in vivo voltammetry (Gratton and Wise, 1994; Kiyatkin, 1994; Kiyatkin and Stein, 1995) are consistent with the findings that dopamine levels are elevated at the onset of cocaine or heroin self-administration, are maintained at a plateau during the period of drug availability, and fall back to baseline when the lever is made inaccessible or during extinction when drug reward is terminated. Voltammetric studies suggest, however, that increases in dopamine concentration precede each injection and that, during the maintenance phase, at least, each injection momentarily drives dopamine levels back down. The interpretation from the voltammetry studies would be that the important factor determining the pattern of DA fluctuations is the level of anticipation of the to-be-earned drug reward and that the fluctuations observed with the microdialysis method are simply delayed reflections of increases that occur before the lever press but show up in the dialysis record 1-2 min later. This interpretation of the cocaine data would suggest approximately a 3 min delay between the actual changes in nucleus accumbens dopamine and the appearance of elevated dopamine in the dialysis recovery cup. In the case of amphetamine, however, in which the mean time between responses is 20 rather than 5 min and in which peak dopamine levels were reached $10-15$ rather than 3 min after each lever press, this explanation clearly fails. The delay between dopamine elevations in nucleus accumbens and dopamine elevations in our dialysis collection vials has been estimated (Wise et al., 1995b) to be $<1 \mathrm{~min}$. For the phasic elevations in dopamine 


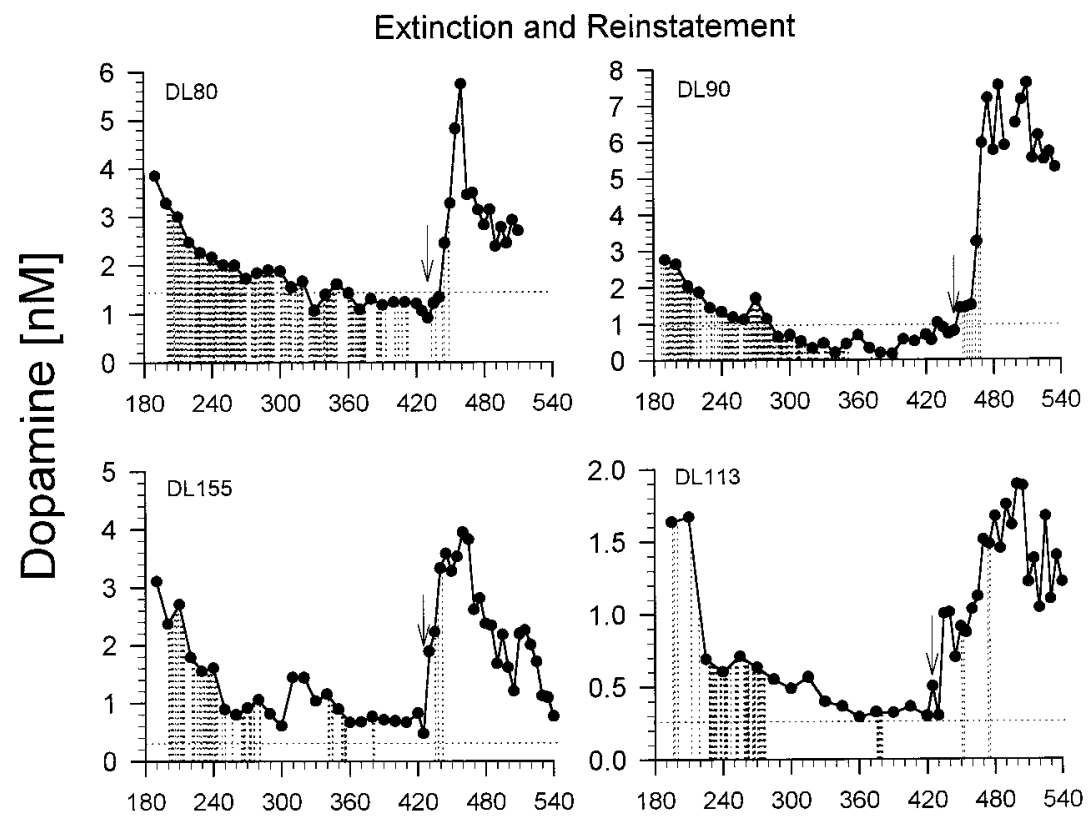

Time [min]
Figure 4. Nucleus accumbens dopamine concentrations in rats lever pressing during a $3 \mathrm{hr}$ extinction period (no drug available) and a $2 \mathrm{hr}$ reinstatement period (drug available). The arrow in each graph represents the time at which a rat received a noncontingent (priming) injection of D-amphetamine and drug was made available again. The horizontal dotted line represents the baseline DA concentration. Dialysate samples were collected at 10 min intervals during the extinction period and at 5 min intervals during the reinstatement period. $D L$ numbers are rat identification numbers.

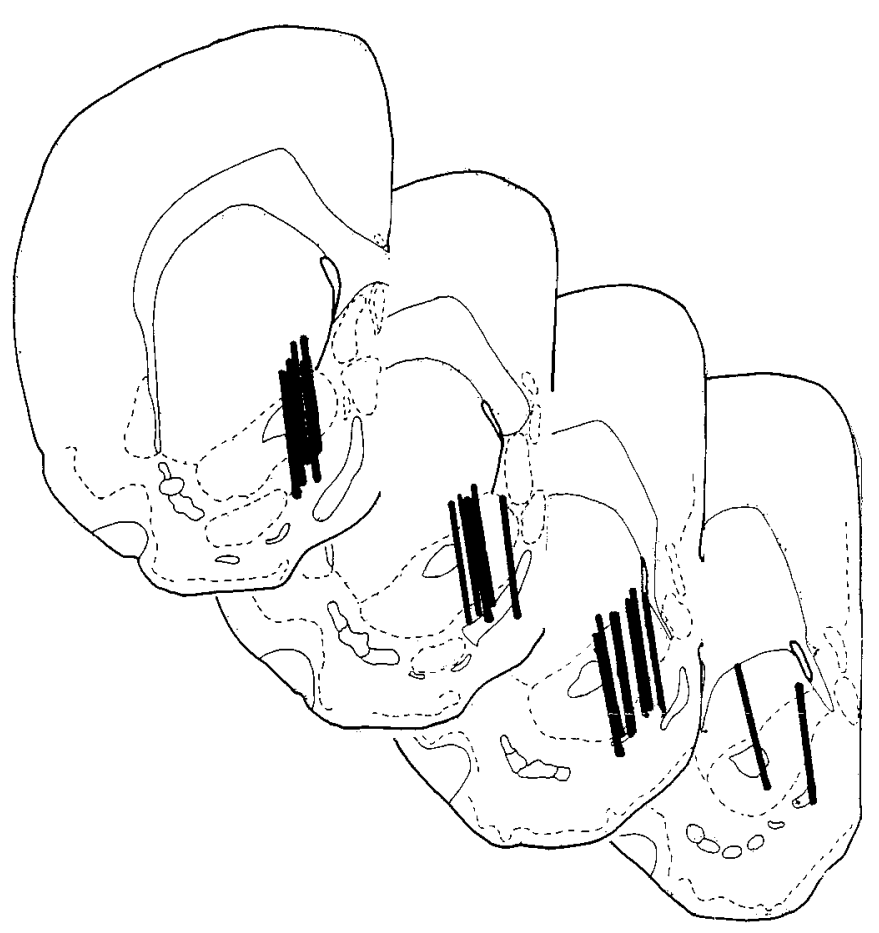

Figure 5. Brain sections taken through the nucleus accumbens showing the locations of microdialysis probes (solid bars) for each of the rats tested here. Drawings are adapted from Pellegrino et al. (1979).

level seen in the present study to reflect actual elevations before the lever press would require a $10 \mathrm{~min}$ or longer delay between dopamine release in the nucleus accumbens and detection of this release in our dialysates. This argument is clearly untenable; although the temporal resolution of microdialysis might be argued to be inadequate to track the 5 min fluctuations of dopamine level caused by fast-acting cocaine, it is clearly adequate to track the 30 min fluctuations of the slower-acting amphetamine.
Further evidence of this point is the fact that similar afterinjection increases in dopamine were seen in yoked injections and after priming injections. It is difficult to imagine how in these cases the observed increases in dopamine level might reflect anticipation of amphetamine reward. The timing of the yoked injections was variable and determined independently from the animals' ongoing behavior; the timing of the priming injections was not linked to any immediate signal that the animals could have identified. Thus the yoked animals and the reinstatement animals could not accurately predict the time of injection and thus would have no signal for anticipatory dopamine release. Thus, evidence that dopaminergic neurons do respond briefly in response to reward-predicting stimuli (Schultz et al., 1992) notwithstanding, the phasic increases in the present study seem clearly to reflect a dominant effect of the receipt and not the anticipation of the drug reward.

These data confirm that self-administered doses of amphetamine are sufficient to elevate dopamine levels in the nucleus accumbens, a finding that is consistent with the long-accepted assumption from pharmacological blockade (Yokel and Wise, 1975) and lesion (Lyness et al., 1979) studies that amphetamine is rewarding because it elevates nucleus accumbens dopamine levels (Fibiger, 1978; Wise, 1978; Beninger, 1983; Bozarth and Wise, 1986). The fact that the animals responded much more in extinction, when dopamine levels were falling to baseline, than they did when drug was available and response rates were only approximately two or three per hour makes it clear that dopamine levels were not driven as a simple or primary correlate of the initiation of instrumental behavior itself. Thus the fluctuations in dopamine level that were predictable from the acute actions and pharmacokinetics of amphetamine primarily overshadowed any fluctuations associated with the initiation of voluntary movement.

In the present experiment, unearned, unexpected (yoked) injections caused elevations in dopamine that were somewhat greater than those caused by self-administered injections. These data are in contrast with those of Hemby and colleagues, who have reported (Hemby et al., 1997) that self-administered cocaine 
caused approximately double the elevation in dopamine levels that was produced by yoked control injections. On the other hand, Hemby et al. (1995) reported the opposite finding for selfadministered and experimenter-administered heroin; in this case they saw dramatic elevations in dopamine levels after unexpected, unearned injections but no significant elevation in dopamine levels after self-administered heroin. The latter finding is difficult to reconcile with the significant elevations in dopamine after self-administered heroin reported by Wise et al. (1995a). Whatever the explanation of these differences, significant elevations were seen with both earned and yoked injections in the present study.

The self-administration of amphetamine at the dose tested here is marked by two phases of drug taking; the response rate in these two phases is under markedly different controls. In the first several minutes of each session, sometimes termed the loading phase, response rates are high and often lack the characteristic pauses between injections that characterize the later phase of the session. Not surprisingly, the present study reveals that dopamine levels soar during the loading phase. This is presumably a period in which the animal finds successive injections additionally rewarding and is, in essence, a phase in which the blood is being loaded with the drug. After the loading phase, the animals cease responding for as long as 1-2 hr, allowing the initial peak in amphetamine concentration to decline significantly (Yokel and Pickens, 1973, 1974).

As the blood amphetamine level falls, the animal eventually resumes lever pressing in what is termed the maintenance phase of the self-administration session; in this phase the animal responds regularly, and amphetamine intoxication is maintained, although at a considerably lower level than was reached at the peak of the loading phase. During the maintenance phase, responses are regularly spaced and serve to maintain blood amphetamine levels above a concentration of $\sim 0.2 \mu \mathrm{g} / \mathrm{ml}$ (Yokel and Pickens, 1974). The animals are capable of lever pressing in the interval between earned injections and can be shown to do so for an alternate reinforcer (Wise et al., 1977), eliminating the possibility that the behavioral stereotypy normally seen during this period severely incapacitates the animal. Animals given a twolever choice between high and low doses adjust their intake by taking the high dose infrequently or the low dose frequently, but they prefer the lever that is associated with the high dose in the case of monkeys (Iglauer et al., 1975) and are more concerned with position than dose in the case of rats (Yokel, 1987); if higher amphetamine concentrations were aversive, they would be expected to learn to choose more-frequent low doses rather than to show a preference for less-frequent high doses. Thus the spaced responding typical of animals in the maintenance phase would not seem to result from aversive consequences of amphetamine concentrations higher than the $0.2 \mu \mathrm{g} / \mathrm{ml}$ concentration at which responding is typically triggered. In our view the most likely explanation of the spaced responding in the maintenance phase is that amphetamine adds no immediate increment in reward to when amphetamine levels-and brain dopamine levels-are elevated beyond the animal's trigger point. In this view, the peak levels reached in the loading phase reflect the fact that the animals are able to respond several times before sensing the full consequences of their recent injections.

The present data make clear that both self-administered and experimenter-administered doses of amphetamine are sufficient to elevate dopamine levels significantly in both naïve and welltrained animals, even when dopamine levels are elevated signifi- cantly by previous amphetamine injections. They demonstrate that dopamine levels fluctuate phasically around the times of lever presses, rising to peak levels after an injection and falling to relatively constant low points just before the next lever press. The extent and course of these phasic fluctuations are arguably attributable to the receipt, and not the anticipation, of drug reward. Finally, these data suggest that, although the direct neurochemical effects of amphetamine on the dopamine system are different from those of cocaine, the mechanisms of action of both psychostimulants as rewarding stimuli appear to be similar and involve nucleus accumbens dopamine.

\section{REFERENCES}

Beninger RJ (1983) The role of dopamine in locomotor activity and learning. Brain Res Rev 6:173-196.

Berger P, Elsworth JD, Reith MEA, Tanen D, Roth RH (1990) Complex interaction of cocaine with the dopamine uptake carrier. Eur J Pharmacol 176:251-252.

Bozarth MA, Wise RA (1986) Involvement of the ventral tegmental dopamine system in opioid and psychomotor stimulant reinforcement. In: Problems of drug dependence, National Institute on Drug Abuse Res Monogr 67 (Harris LS, ed), pp 190-196. Washington, DC: US Government Printing Office.

Dougherty J, Pickens R (1974) Effects of phenobarbital and SKF 525A on cocaine self-administration in rats. In: Drug addiction, Vol 3, Neurobiology and influences on behavior (Singh JM, Lal H, eds), pp 135-143. Miami: Symposia Specialists.

Fibiger HC (1978) Drugs and reinforcement mechanisms: a critical review of the catecholamine theory. Annu Rev Pharmacol Toxicol 18:37-56.

Gerber GJ, Wise RA (1989) Pharmacological regulation of intravenous cocaine and heroin self-administration in rats: a variable dose paradigm. Pharmacol Biochem Behav 32:527-531.

Gratton A, Wise RA (1994) Drug- and behavior-associated changes in dopamine-related electrochemical signals during intravenous cocaine self-administration in rats. J Neurosci 14:4130-4146.

Heikkila RE, Orlansky H, Cohen G (1975a) Studies on the distinction between uptake inhibition and release of $(3 \mathrm{H})$ dopamine in rat brain tissue slices. Biochem Pharmacol 24:847-852.

Heikkila RE, Orlansky H, Mytilineou H, Cohen G (1975b) Amphetamine: evaluation of $\mathrm{D}$ - and $\mathrm{L}$-isomers as releasing agents and uptake inhibitors for $3 \mathrm{H}$-dopamine and $3 \mathrm{H}$-norepinephrine in slices of rat neostriatum and cerebral cortex. J Pharmacol Exp Ther 194:47-56.

Hemby SE, Martin TJ, Co C, Dworkin SI, Smith JE (1995) The effects of intravenous heroin administration on extracellular nucleus accumbens dopamine concentrations as determined by in vivo microdialysis. J Pharmacol Exp Ther 273:591-598.

Hemby SE, Co C, Koves TR, Smith JE, Dworkin SI (1997) Differences in extracellular dopamine concentrations in the nucleus accumbens during response-dependent and response-independent cocaine administration in the rat. Psychopharmacology (Berl) 133:7-16.

Iglauer C, Lewellyn ME, Woods JH (1975) Concurrent schedules of cocaine injection in rhesus monkeys: dose variations under independent and non-independent variable-interval procedures. Pharmacol Rev 27:367-383.

Kiyatkin EA (1994) Changes in dopamine-dependent electrochemical signal in the nucleus accumbens associated with repeated cocaine injections in rats. Brain Res 642:228-236.

Kiyatkin EA, Stein EA (1995) Fluctuations in nucleus accumbens dopamine during cocaine self-administration behavior: an in vivo electrochemical study. Neuroscience 64:599-617.

Lyness WH, Friedle NM, Moore KE (1979) Destruction of dopaminergic nerve terminals in nucleus accumbens: effect on D-amphetamine self-administration. Pharmacol Biochem Behav 11:553-556.

Pellegrino LJ, Pellegrino AS, Cushman JA (1979) A stereotaxic atlas of the rat brain. New York: Plenum.

Pickens R, Thompson T (1968) Cocaine reinforced behavior in rats: effects of reinforcement magnitude and fixed-ratio size. J Pharmacol Exp Ther 161:122-129.

Pickens R, Thompson T (1971) Characteristics of stimulant drug reinforcement. In: Stimulant properties of drugs (Thompson T, Pickens R, eds), pp 177-192. New York: Appleton-Century-Crofts.

Robinson TE, Whishaw IQ (1988) Normalization of extracellular dopa- 
mine in striatum following recovery from a partial unilateral 6-OHDA lesion of the substantia nigra: a microdialysis study in freely moving rats. Brain Res 450:209-224.

Schultz W (1997) A neural substrate of prediction and reward. Science 275:1593-1599.

Schultz W, Apicella P, Scarnati E, Ljungberg T (1992) Neuronal activity in monkey ventral striatum related to the expectation of reward. J Neurosci 12:4595-4610.

Wise RA (1978) Catecholamine theories of reward: a critical review. Brain Res 152:215-247.

Wise RA (1993) In vivo estimates of extracellular dopamine and dopamine metabolite levels during intravenous cocaine or heroin selfadministration. Semin Neurosci 5:337-342.

Wise RA, Bozarth MA (1987) A psychomotor stimulant theory of addiction. Psychol Rev 94:469-492.

Wise RA, Yokel RA, Hansson P, Gerber GJ (1977) Concurrent intracranial self-stimulation and amphetamine self-administration in rats. Pharmacol Biochem Behav 7:459-461.

Wise RA, Leone P, Rivest R, Leeb K (1995a) Elevations of nucleus accumbens dopamine and DOPAC levels during intravenous heroin self-administration. Synapse 21:140-148.

Wise RA, Newton P, Leeb K, Burnette B, Pocock D, Justice Jr JB (1995b) Fluctuations in nucleus accumbens dopamine concentration during intravenous cocaine self-administration in rats. Psychopharmacology (Berl) 120:10-20.

Yokel RA (1987) Intravenous self-administration: response rates, the effects of pharmacological challenges, and drug preferences. In: Methods of assessing the reinforcing properties of abused drugs (Bozarth MA, ed), pp 1-33. New York: Springer.

Yokel RA, Pickens R (1973) Self-administration of optical isomers of amphetamine and methylamphetamine by rats. J Pharmacol Exp Ther 187:27-33.

Yokel RA, Pickens R (1974) Drug level of D- and L-amphetamine during intravenous self-administration. Psychopharmacology (Berl) 34:255-264.

Yokel RA, Wise RA (1975) Increased lever pressing for amphetamine after pimozide in rats: implications for a dopamine theory of reward. Science 187:547-549. 